\title{
Exposure to a fearful context during periods of memory plasticity impairs extinction via hyperactivation of frontal-amygdalar circuits
}

\author{
James M. Stafford, DeeAnna K. Maughan, Elena C. Ilioi, and K. Matthew Lattal ${ }^{1}$ \\ Department of Behavioral Neuroscience, Oregon Health \& Science University, Portland, Oregon 97239-3098, USA
}

\begin{abstract}
An issue of increasing theoretical and translational importance is to understand the conditions under which learned fear can be suppressed, or even eliminated. Basic research has pointed to extinction, in which an organism is exposed to a fearful stimulus (such as a context) in the absence of an expected aversive outcome (such as a shock). This extinction process results in the suppression of fear responses, but is generally thought to leave the original fearful memory intact. Here, we investigate the effects of extinction during periods of memory lability on behavioral responses and on expression of the immediate-early gene c-Fos within fear conditioning and extinction circuits. Our results show that long-term extinction is impaired when it occurs during time periods during which the memory should be most vulnerable to disruption (soon after conditioning or retrieval). These behavioral effects are correlated with hyperactivation of medial prefrontal cortex and amygdala subregions associated with fear expression rather than fear extinction. These findings demonstrate that behavioral experiences during periods of heightened fear prevent extinction and prolong the conditioned fear response.
\end{abstract}

[Supplemental material is available for this article.]

One of the major theoretical challenges in basic research on the neurobiology of memory is to determine the conditions in which memories can be suppressed, or even erased. This has implications not just for understanding basic mechanisms of memory, but also for applications that translate basic research into treatments that may improve clinical options for disorders that involve a debilitating inability to suppress invasive, fearful memories. Many studies dating back to Pavlov (1927) have demonstrated that exposure to previously conditioned stimuli can eliminate behavioral responses, but these studies have consistently found that this extinction process suppresses the original memory without eliminating that memory (e.g., Bouton 2004; Delamater 2004).

Several recent studies of memory have asked whether extinction under certain conditions can erase, rather than just suppress, previously established memories (Myers et al. 2006; Norrholm et al. 2008; Schiller et al. 2008; Monfils et al. 2009). One popular idea is that extinction during periods of memory lability, which is thought to occur soon after acquisition or retrieval ("immediate extinction"), may permanently displace the original fear memory with a new, safe memory. Evidence for this idea, however, is mixed, with some studies showing no effect or impairments in extinction when it occurs soon after acquisition or retrieval of fear (Morris et al. 2005; Chan et al. 2010; Kindt and Soeter 2013).

A key to determining the effectiveness of an extinction session may be to determine the conditions under which neurobiological circuitry associated with long-term extinction is engaged. Many studies have demonstrated that when extinction occurs at least $24 \mathrm{~h}$ after initial conditioning ("delayed extinction"), crosstalk between the amygdala and prefrontal cortex mediates lasting changes in performance (Quirk and Mueller 2008; Li et al. 2011). In addition, studies of the amygdala and medial prefrontal cortex have begun to show some of the substrates that underlie immedi-

\footnotetext{
1'Corresponding author

E-mail lattalm@ohsu.edu

Article is online at http://www.learnmem.org/cgi/doi/10.1101//m.029801.112.
}

ate extinction (Mao et al. 2006; Clem and Huganir 2010; Kim et al. 2010; Xue et al. 2012). One brain region that is strongly linked with the prefrontal cortex and controls different aspects of fear expression and extinction is the intercalated cell masses (ITC) within the amygdala (Hefner et al. 2008; Likhtik et al. 2008; Busti et al. 2011; Manko et al. 2011). However, little is known about how these specific neuronal populations may be engaged in response to extinction contingencies that result in differential response loss (e.g., immediate or delayed extinction).

To date, no study has compared the effects of extinction soon after conditioning with those effects soon after retrieval. To examine this we conducted extinction at post-acquisition and postretrieval windows that have previously been shown to be within periods of memory lability (Fig. 1; Bourtchouladze et al. 1998; Monfils et al. 2009; Stafford and Lattal 2009). Second, we examine changes in the product of the immediate early gene, c-Fos, induced by these treatments. We find that across a variety of behavioral conditions, extinction soon after acquisition or retrieval prevents the retention of extinction during subsequent test sessions. These effects correspond to differential responses in the prelimbic cortex and in the subpopulations of the amygdala (e.g., ITC, BA, CeA).

\section{Results}

Experiment 1. Effect of post-acquisition delay on extinction of fear expression and sensitivity to extinction

\section{Effect of acquisition to extinction interval}

Both groups receiving a shorter delay between acquisition and extinction $(0 \mathrm{~h}$ and $1 \mathrm{~h}$ ) showed significantly more spontaneous recovery than those receiving a longer delay between acquisition and extinction ( 4 and $24 \mathrm{~h}$ ) during the first $3 \mathrm{~min}$ of the 1 -d test (effect of extinction recency) (Fig. 2A). A main effect of extinction recency $\left(F_{(3,48)}=2.94, P=0.048\right)$ followed by post hoc confirmed 


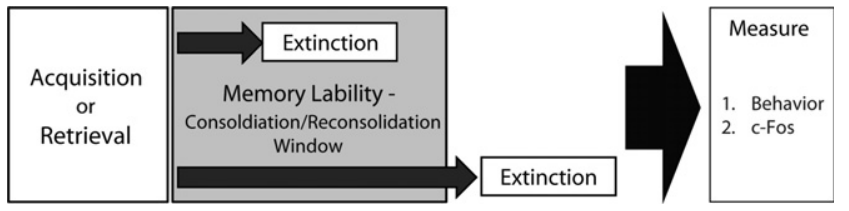

Figure 1. General experimental design. Contextual fear extinction occurred inside or outside of previously demonstrated post-acquisition or post-retrieval periods of memory vulnerability. Behavior was tested and c-Fos immunohistochemistry was examined following extinction.

this as both the 24-h and 4-h groups froze less than either 1-h or 0-h groups (all $P<0.05$ ). Importantly, mice were brought to similar levels of performance by the end of the extinction session, as revealed through short-term tests (Ext-Imm and 1-d Imm, all $P>$ 0.1; Supplemental Fig. 1A). A follow-up experiment showed that even a very remote $(50 \mathrm{~d})$ interval between acquisition and extinction produced more robust behavioral extinction compared to extinction immediately following acquisition (Supplemental Fig. 1B). This study shows that long delays between acquisition and extinction, particularly those outside the hypothesized consolidation window, attenuate spontaneous recovery more strongly than do short acquisition-extinction intervals.

\section{Sensitivity of immediate or delayed extinction to extinction duration}

The robust attenuation of spontaneous recovery with a 24-h acquisition-extinction delay extended to mice receiving strong extinction ( $24 \mathrm{~min}$ ) but not weak extinction ( $3 \mathrm{~min}) 24 \mathrm{~h}$ following acquisition (Fig. 2B). Prior to test, mice were brought to common levels of performance by the end of the extinction sessions (Supplemental Fig. 1C; all $P$ 's $>0.1$ ). A main effect of extinction recency during the $3 \mathrm{~min}$ of Test $1\left(F_{(1,32)}=5.7, P=0.024\right)$ followed by post hoc indicated that the mice receiving strong extinction ( $24 \mathrm{~min})$ at a 24 -h delay froze significantly less than those receiving extinction immediately following acquisition $(P=0.02)$. This further replicated the basic finding that immediate extinction produces more spontaneous recovery and revealed that mice were insensitive to the duration of the extinction session when extinction occurred immediately after acquisition or retrieval.

\section{Experiment 2. Acquisition-extinction interval: Vulnerability to spontaneous recovery}

Testing following extinction revealed that immediate extinction left behavior more vulnerable to spontaneous recovery even when mice were brought to the same levels of performance following extinction and each test day (Fig. 3). A time $\times$ extinction recency interaction confirmed that mice receiving immediate or $24-\mathrm{h}$ extinction were brought to the same levels of performance at the end of extinction and each test $(P>0.1$ in the final time block of each session). A significant effect of extinction recency during the first $3 \mathrm{~min}$ of the first three tests indicates that mice receiving extinction $24 \mathrm{~h}$ following acquisition froze significantly less than those receiving immediate extinction (all $t>2, P<0.04$ ). Test 4 and the test following the 14-d retention interval (18 d) showed strong trends toward less recovery in the 24-h extinction group $(P=0.09$ and $P=0.055$, respec$P<0.05$. tively). The critical finding from this experiment is that the timing of extinction relative to acquisition is a critical determinant of subsequent spontaneous recovery as immediate extinction produced greater recovery even when mice received repeated extinction (test) sessions.

Experiment 3. Context processing in immediate extinction Mice receiving extinction immediately following acquisition showed decreased freezing relative to a group that received no extinction (No Ext) or extinction outside the conditioning/testing context (Imm-B) when tested $1 \mathrm{~d}$ later in the same context they received extinction (Imm-A) (Fig. 4). A main effect of extinction treatment confirmed a difference between groups $\left(F_{(2,57)}=7.9\right.$, $P=0.001)$. Post hoc indicated that, indeed, the Imm-A group froze less than the No Ext and Imm-B group $(P<0.001$ and $P=$ 0.035 , respectively). This effect was not due to differences in baseline levels of performance as mice undergoing nonreinforced context exposure in Context $\mathrm{A}$ and Context $\mathrm{B}$ were brought to similar levels of performance by the end of the extinction day $(P>0.1)$. This study shows that the greater spontaneous recovery seen in immediate extinction is not due to a failure to extinguish or process the extinction context.

\section{Experiment 4. Effect of post-retrieval delay on extinction of fear expression and sensitivity to extinction}

\section{Effect of retrieval-to-extinction interval}

A 24-h delay between fear memory retrieval and extinction produces more robust and persistent extinction (1- and 14-d test) than do shorter delays (Fig. 5A). A significant main effect of extinction recency $\left(F_{(3,28)}=4.1, P=0.016\right)$ followed by post-hoc analysis revealed that, indeed, the 24 -h groups froze less than the 0 -h group on the 1 -d test $(P=0.003)$. When tested $14 \mathrm{~d}$ later, a 24 -h post-retrieval interval induced significantly less freezing than a 0 -h interval $(P=0.044)$. Differences on test were not due to differences in performance prior to testing as all groups showed similar levels of freezing during both retrieval and the last block of extinction (Supplemental Fig. 2A; all $P>0.1$ ).

\section{Sensitivity of immediate or delayed extinction to extinction duration}

A long extinction session $24 \mathrm{~h}$ following retrieval produced more robust extinction compared to a session soon after retrieval (Fig. 5B). Extinction recency had a significant effect on freezing during the first test. This was driven by the 24-h 24-min group freezing significantly less than groups receiving a 0 -h post-extinction delay
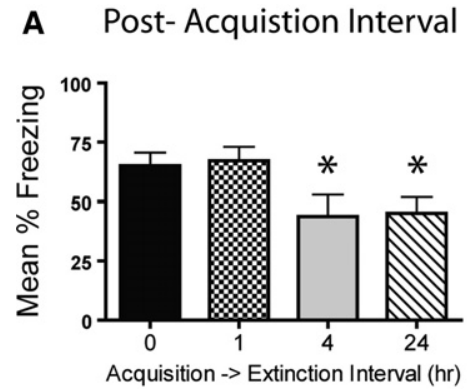

B Extinction Strength

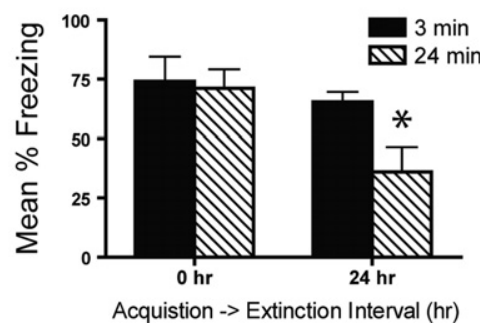

Figure 2. Extinction immediately following acquisition impairs extinction and decreases sensitivity to extinction strength. $(A)$ Extinction at short post-acquisition delays caused less robust response loss than did that at longer delays ( $24 \mathrm{~h}$ ) when tested $1 \mathrm{~d}$ after extinction. (B) Mice receiving extinction immediately following acquisition were insensitive to extinction strength ( 3 or $24 \mathrm{~min}$ ), whereas mice receiving extinction at a 24 -h delay showed more robust response loss in the presence of strong extinction. $\left(^{*}\right)$ 


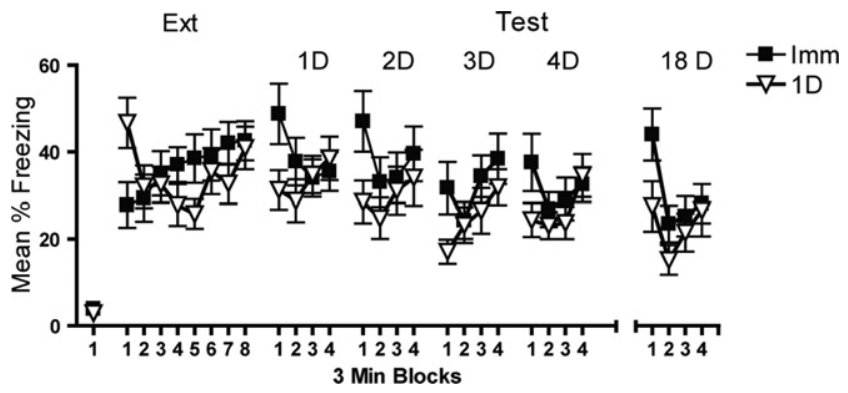

Figure 3. Extinction immediately following acquisition leaves behavior more vulnerable to spontaneous recovery than delayed extinction. Mice were brought to similar levels of performance following extinction and test. However, mice were more vulnerable to spontaneous recovery if extinction occurred immediately $(0 \mathrm{~h})$ rather than $1 \mathrm{~d}$ following acquisition.

regardless of extinction duration (all $P<0.01$ ) or the group receiving 3 min extinction at a $24-\mathrm{h}$ interval $(P=0.003)$. A similar effect was seen at the 14-d test with a main effect of recency $\left(F_{(3,28)}=\right.$ $3.9, P=0.02)$ and the 24-h 24-min extinction group freezing significantly less than the 0 -h 3 -min group $(P=0.03)$ and the 0 -h 24 -min group $(P=0.05)$. Importantly, mice in immediate and delayed groups did not differ in performance during extinction regardless of extinction duration (Supplemental Fig. 2B; all $P$ 's $P>$ 0.05). Like Experiments 1 and 2, these results indicate that a short retrieval-extinction interval led to greater spontaneous recovery and decreased sensitivity to extinction strength.

\section{Immediate extinction deficit depends on memory retrieval}

Attenuated spontaneous recovery was seen only at 1- and 14-d tests if retrieval preceded delayed extinction $(24 \mathrm{~h})$, but not if retrieval was absent or if extinction occurred immediately following retrieval (Fig. 5C). On both the 1- and 14-d tests, a main effect of recency retrieval conditions $\left(F_{(3,24)}=3.8, P=0.038\right)$ followed by post hoc revealed that extinction $24 \mathrm{~h}$ following retrieval resulted in less freezing than any group that did not receive retrieval prior to extinction (all $P<0.05$ ) or the group that received extinction immediately following retrieval $(P<0.03)$. A similar effect was found at the 14-d test with the main effect of recency retrieval conditions $\left(F_{(3,22)}=2.3, P=0.014\right)$ being driven by extinction $24 \mathrm{~h}$ following retrieval resulting in significantly less freezing than the group receiving extinction in the absence of retrieval $(P=0.031)$. A baseline difference at the end of extinction (Supplemental Fig. 2C; $P<0.02$ ) may contribute to differences observed during tests. However, the main effect of the interval between retrieval and extinction has been repeatedly replicated without this baseline difference (Experiment 4) making it unlikely that this difference significantly confounds the effects of this experiment.

\section{Experiment 5. Immunohistochemistry}

\section{mPFC}

Immediate extinction following both acquisition and retrieval strongly activates the prelimbic (PrL) cortex (Fig. 6; main effect of extinction recency, $\left.F_{(1,27)}=9.7, P=0.004\right)$. Mice receiving fear conditioning in the absence of extinction did not display elevated PrL c-Fos, indicating that this effect was contingent on extinction delay rather than simply activation post-acquisition.

No consistent effect of extinction delay on c-Fos in the IL cortex was seen. A significant interaction between extinction recency $\times$ pre-extinction conditions (fear acquistion or retrieval) $\left(F_{(1,27)}=6.9, P<0.014\right)$ followed by post hoc revealed that the only simple main effect was with the immediate acquisition group showing greater c-Fos expression than the delayed acquisition group $(P=0.014)$.

Amygdala

Activation within amygdala subregions varied across immediate and delayed extinction treatments, with some regions showing greater activation following immediate extinction (e.g., BLA, $\mathrm{CeA}$ ) while others showed the opposite with patterns that depended on relative activation in related nuclei (e.g., ITC) (Fig. 7).

\section{Basolateral amygdala}

Within the BLA, an extinction recency $\times$ pre-extinction conditions interaction $\left(F_{(1,26)}=5.28, P=0.03\right)$ with planned comparison follow-up indicates that immediate extinction induced greater c-Fos than delayed extinction (24-h group; all $P \leq 0.05$ ). The interaction was driven by the immediate acquisition group displaying greater activation than the immediate retrieval group $(P=0.03)$. The No Ext group differed only from the delayed groups (all $P^{\prime} \mathrm{s}<0.01$ ).

\section{Central nucleus}

Within the central lateral nucleus, a main effect of extinction recency $\left(F_{(1,27)}=11.1, P=0.003\right)$ revealed that immediate extinction induced greater c-Fos expression than delayed extinction. Interestingly, there was no difference between the No Ext group and the delayed extinction groups, indicating that this effect was not generally due to shock prior to c-Fos quantification. In contrast, a main effect of recency $\left(F_{(1,27)}=8.1, P=0.01\right)$ and preextinction conditions $\left(F_{(1,27)}=6.0, P=0.02\right)$ within the central medial nucleus $(\mathrm{CeM})$ indicates that while immediate extinction produced greater c-Fos activation, so did being shocked prior to extinction. The main effect of pre-extinction conditions (fear acquisition or retrieval) was likely due to shock immediately prior to extinction as the No Ext group also showed Fos levels above those of the delayed group $(P<0.001)$.

\section{Intercalated cells}

Detailed analysis of the intercalated cells of the amygdala revealed that the proportion of main nucleus (In) c- $\mathrm{Fos}^{+}$neurons relative to medial paracapsular (ImP) neurons was greater in mice receiving extinction $24 \mathrm{~h}$ following acquisition/retrieval than in mice receiving immediate extinction (Fig. 7). This was confirmed by a main effect of extinction recency $\left(F_{(1,26)}=40, P<0.001\right)$ and no

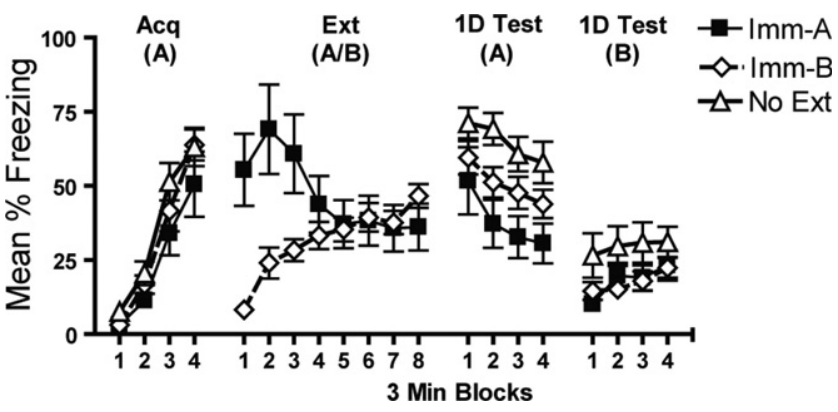

Figure 4. Immediate extinction does not produce a failure to process the extinction context. Mice receiving extinction immediately following acquisition showed decreased freezing relative to a group that received no extinction (No Ext) or extinction outside the conditioning/testing context (Imm-B) when tested $1 \mathrm{~d}$ later in the same context they received extinction (Imm-A). 
A Post- Retrieval Interval

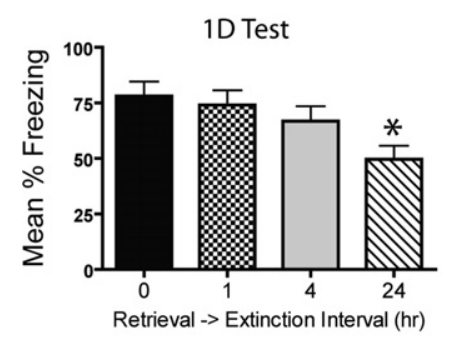

\section{B Extinction Strength 1D Test}

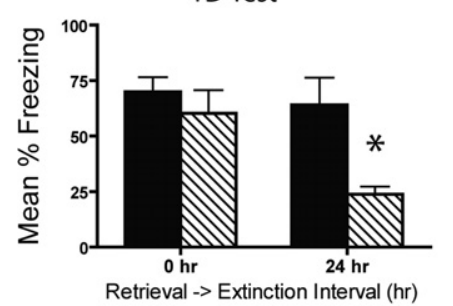

C Effect of Retrieval

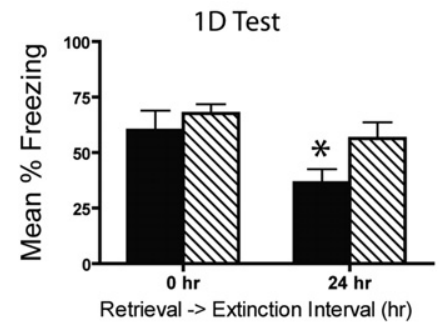

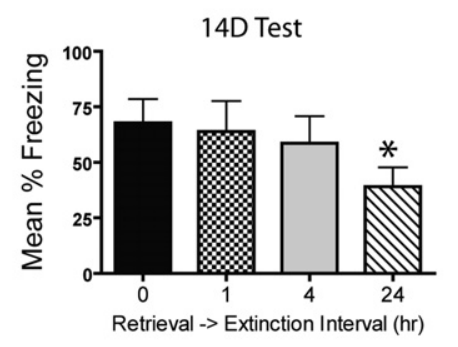
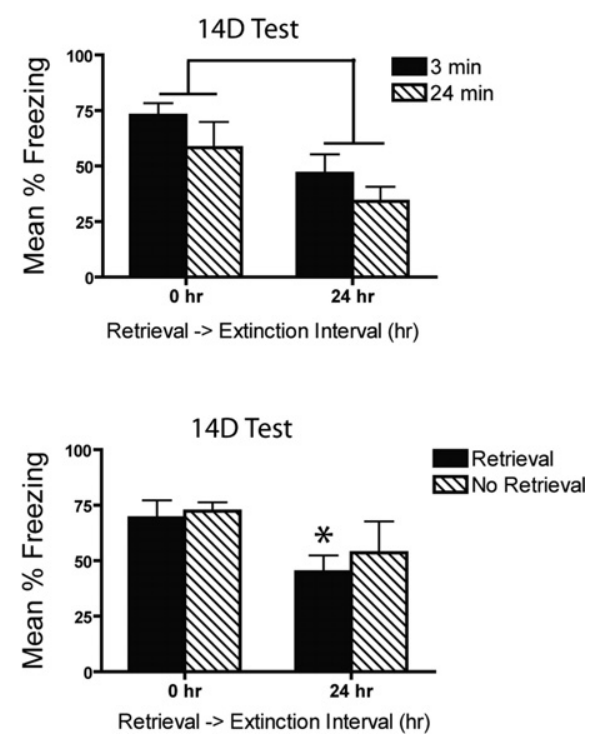

Figure 5. A short retrieval to extinction interval produces lasting impairments in extinction and decreases sensitivity to extinction strength. (A) Extinction 0-h post-retrieval caused less robust response loss than did longer delays ( $24 \mathrm{~h}$ ) when tested 1 and $14 \mathrm{~d}$ after extinction. (B) Mice receiving extinction immediately following acquisition were insensitive to extinction strength (3 or $24 \mathrm{~min}$ ), whereas mice receiving extinction at a 24-h delay showed more robust response loss in the presence of strong extinction. $\left(^{*}\right) P<0.05$. (C) Attenuated spontaneous recovery was only seen at 1 - and $14-d$ tests if retrieval preceded delayed extinction $(24 \mathrm{~h})$, but not if retrieval was absent or if extinction occurred immediately following retrieval. (*) $0 \mathrm{hr}$ vs. $24 \mathrm{hr}, P<0.05$.

significant extinction recency $\times$ pre-extinction conditions interaction. Furthermore, the No Ext groups significantly differed from both the immediate and delayed extinction groups $(P=$ 0.024 and $P<0.001$, respectively). When evaluating the subpopulations alone, the consistent finding is that shock strongly activates both intercalated cell masses, with greater c-Fos expression in the immediate acquisition and No Ext groups (all $P<0.05$ ).

Combined with the behavioral data, the IHC suggests that the activity of the In relative to ImP may serve to signal contingencies that result in strong extinction.

\section{Representative c-Fos immunohistochemistry images for each brain region studied are presented in Supplemental Figure 3}

Together these results indicate that hyperactivity in the PrL and select amygdalar subregions (CeA and BA) may underlie a deficit in extinction while the proportion of In to ImP active neurons signals contingencies associated with robust extinction.

\section{Discussion}

The critical finding from these studies was that under a variety of conditions, an extinction session conducted soon after acquisition or retrieval produced poor behavioral extinction, relative to longer delays. These effects were replicated both within and between experiments with consistent results across experiments. Importantly, control experiments showed that poor extinction produced by immediate extinction was not due to failures to learn extinction contingencies and critically depended on the interval between behavioral manipulations under common testing conditions. Immunohistochemistry for the IEG c-Fos revealed that hyperactivity in the prelimbic cortex and the relative activity of In to ImP regions, and other amygdala subregions, contribute to the deficits in extinction after short delays.

Our behavioral results provide new insight into a growing body of literature on the effects of time before extinction following acquisition or retrieval. These findings are consistent with those indicating that extinction shortly after fear learning or fear cue exposure impairs extinction and exacerbates spontaneous recovery, renewal, and reinstatement (Morris et al. 2005; Chan et al. 2010; Costanzi et al. 2011; Ishii et al. 2012). Other studies have found opposing results-that extinction soon after acquisition (Myers et al. 2006) or retrieval (Monfils et al. 2009) promotes the retention of extinction (see Flavell et al. 2011). Some of these differences may be attributed to differences in how behavior is assessed (e.g., change in behavior from extinction to test as in Monfils et al. [2009] or common test performance as in Chan et al. [2010]), when behavior is assessed (Johnson et al. 2010), the type of preparation used (e.g., fear-potentiated startle in Myers et al. [2006] or cued fear conditioning in Maren and Chang [2006]), species used, or the particular intervals used in the different experiments.

However, it is important to note that even when species and experimental paradigms are closely matched, there is still discordance between results (e.g., Monfils et al. 2009; Chan et al. 2010). Further, some studies have shown similar effects to those of Monfils et al. (2009) using contextual fear preparations in mice (Rao-Ruiz et al. 2011) while others show findings like ours in contextual paradigms (Ishii et al. 2012). Although important to study, it is unlikely that the discrepant findings in the literature are simply due to procedural differences, such as the type of fear conditioning (cued or contextual), the postsession delay (immediate or $10 \mathrm{~min}$ ), or differences in species. Our effects were quite similar regardless of whether we were examining extinction after initial conditioning or retrieval. This suggests that similar mechanisms operate, although the effectiveness of the 4-h window in producing extinction in the acquisition experiment, but not in the retrieval experiment suggests a potentially interesting temporal dissociation that is worth further exploration.

Our immunohistochemistry results suggest that immediate extinction - whether it followed conditioning or retrieval - induces high c-Fos expression within the PrL and certain amygdala 


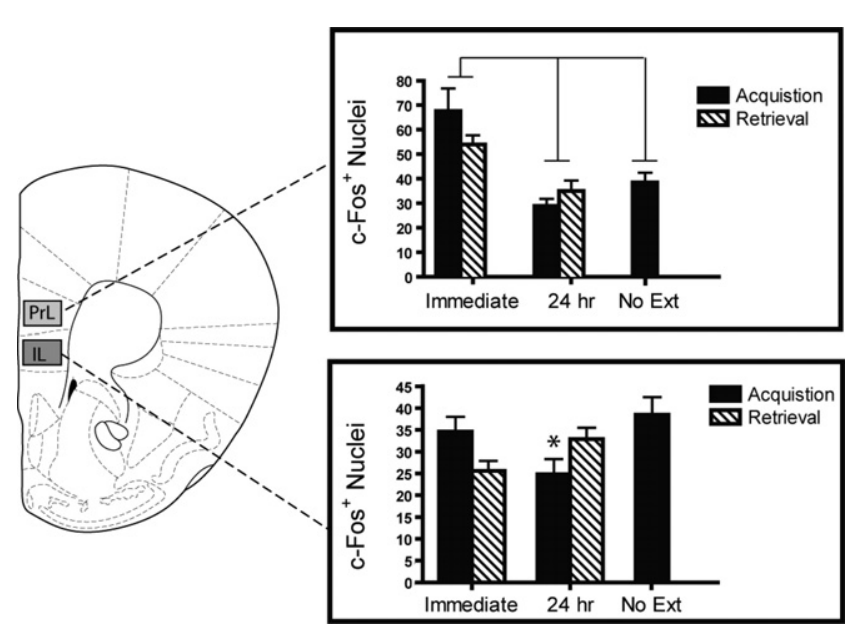

Figure 6. Differential activation of $\mathrm{MPFC}$ is associated with the immediate extinction deficit. Immediate extinction $(0 \mathrm{~h})$ induced strong c-Fos expression in the prelimbic ( $\mathrm{PrL}$ ) cortex compared to delayed extinction $(24 \mathrm{~h})$. Activation within the infralimbic (IL) cortex showed variations in sensitivity to extinction recency. Lines between bars denote $P<0.05$. $\left(^{*}\right)$ Acquisition delay $<$ acquisition immediate, $P<0.05$.

subregions (CeA and BLA), suggesting that hyperactivity in these brain regions is associated with poor extinction retention (Xue et al. 2012, but see Kim et al. 2010). In contrast, delayed extinction-whether it followed conditioning or retrieval-increased the proportion of main nucleus (In) c-Fos ${ }^{+}$neurons relative to medial paracapsular (ImP) neurons. This finding is consistent with studies indicating that activation of these two neuronal populations competes during learning, with the paracapsular cluster (ImP) more active during both acquisition and extinction and the main nucleus (In) activated preferentially during fear extinction (Whittle et al. 2010; Busti et al. 2011; Manko et al. 2011).

These ITC data fit well with our other amygdala data and recent amygdala connectivity studies (for reviews, see Palomares-Castillo et al. 2012; Pare and Duvarci 2012). For example, it has been postulated that the dorsal ImP group drives fear expression states by functionally disinhibiting the CeM via inhibition of CeL "off" neurons while inhibiting the In. Conversely, when not under inhibitory influence from the ImP, the In is thought to drive extinction via direct inhibition of the CeM. Therefore, when there is more $\operatorname{ImP}$ activation relative to the In, the CeM should be more active. In contrast, more relative In activation should result in less CeM activity. This is the pattern seen in our data, with the relatively greater $\mathrm{ImP} /$ greater $\mathrm{CeM}$ activation in the group showing the greatest fear (immediate extinction, No Ext) while relatively greater In/less CeM activity was associated with stronger extinction (delayed extinction). Combined with the hyperactivity seen in the PL and BA when extinction was poor (i.e., immediate), this suggests a complex network that extends from distinct mPFC populations to these very specific amygdala populations in mitigating the effect of extinction recency. However, more work is needed to determine the specificity of these microcircuits as the cell-type (PKC $\delta+/-$; glutamate/GABAergic receptor subtypes) and the precise afferents/efferents of these amygdala subregions are critically important in regulating fear expression and extinction (Ciocchi et al. 2010; Haubensak et al. 2010; Dobi et al. 2013).

The critical theoretical implication of our findings is that extinction during periods when the original fearful memory has been shown to be most vulnerable to pharmacological disruption does not erase or prevent a memory from forming. One would therefore need an account with mechanisms that prevent a labile memory from being updated with the new information that occurs during the extinction trial. Some studies suggest that even minimally invasive procedures during periods of memory consolidation can strengthen the fear response (Hui et al. 2006), as can other behavioral and pharmacological enhancements of the stress response (McGaugh 2006; McGaugh and Roozendaal 2009). Thus, the simple experience of being returned to a fearful context while the organism is in an aroused state may prevent extinction. This is supported by a large body of evidence showing that activating the adrenergic system during memory formation augments amygdala responsivity, leading to more robust emotional memory formation and expression. In fact, this increased amygdala (e.g., CeA/BLA) reactivity is often associated with heightened dorsal prefrontal activity, a finding consistent with our immunohistochemistry data (McIntyre et al. 2012). Our data also demonstrate that expression of fear may be disrupted soon after conditioning, but that short-term extinction (both within-session extinction and short-term memory tests) is not necessarily impaired,

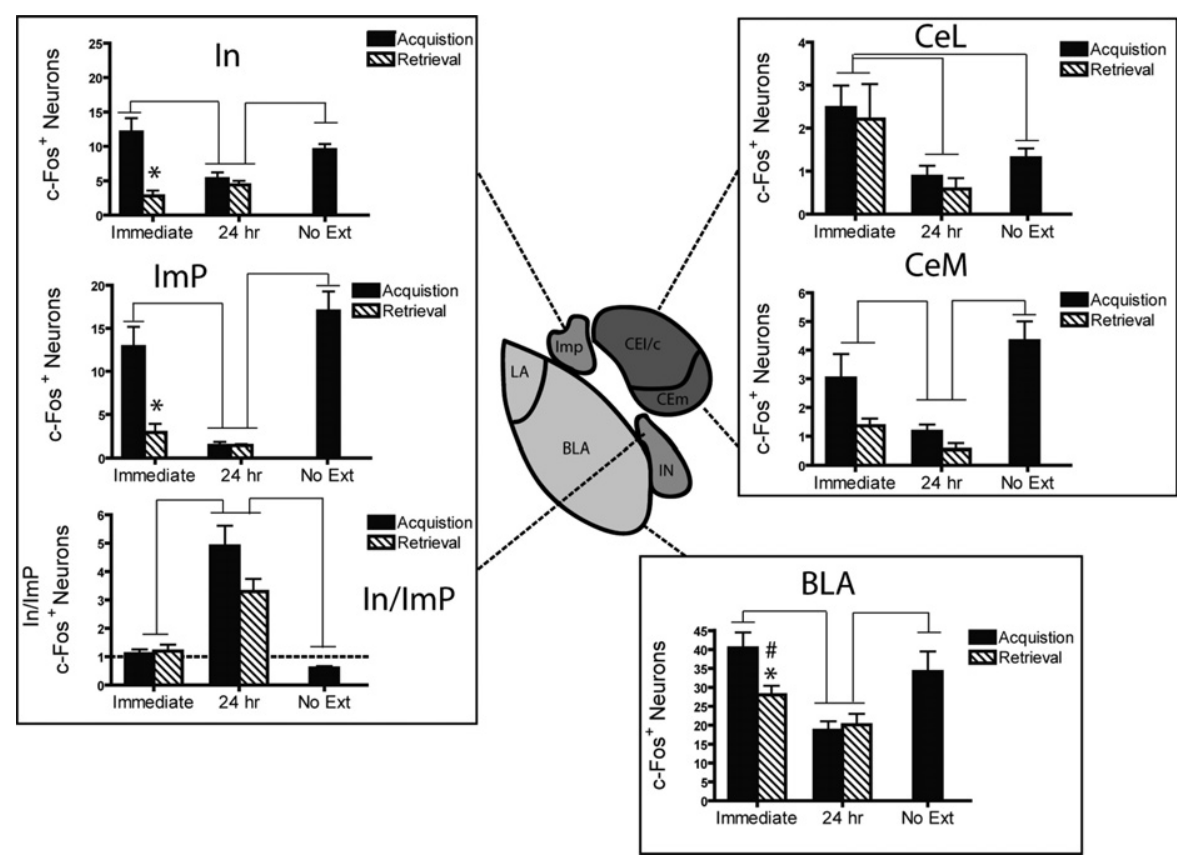

Figure 7. Differential activation of amygdala is associated with the immediate extinction deficit. A 24-h delay between acquisition or retrieval and extinction induced less c-Fos expression in the central (CeL/CeM) and basolateral amygdala (BLA) than did immediate or no extinction. In contrast, delayed extinction induced strong $\ln : \mathrm{ImP}$ activation, the immediate extinction group showed neutral In:ImP activation, and the no extinction group showed an inverse In:ImP ratio. Lines between bars denote $P<0.05$. $\left(^{*}\right)$ Acquisition delay $<$ acquisition immediate, $P<0.05$. 
which is consistent with an effect on consolidation of long-term extinction.

Along the same lines, other accounts suggest that memories in different states of activity will prevent new learning from occurring during extinction. For example, according to sometimes-opponent process (SOP) theory, more of the contextual memory will be in a secondary state of activity during extinction soon after acquisition or retrieval. This will result in less of that contextual representation being retrieved to a primary state of activity during extinction and consequently impair the development of inhibitory learning (see Brandon et al. 2003; Morris et al. 2005). Similar accounts would predict that effects of extinction recency may also be attributable to proactive interference where the first active memory trace (i.e., new fear acquisition or fear retrieval) proactively interferes with the second learning event (i.e., extinction) (Gleitman and Jung 1963).

An account that brings together aspects of each of the aforementioned theories is one alluded to by Chan et al. (2010). This account suggests that animals are sensitive to the differences between the fearful or acquisition state and the extinction state (Capaldi 1966; Redish et al. 2007). In the context of these experiments, when extinction closely follows acquisition or retrieval (which strongly engages the original fearful CS-US memory), the subjects have trouble distinguishing between whether the nonreinforced context/CS exposure during extinction still predicts the original fear contingency. This creates an ambiguous state where the mice are forced to maintain the original fear memory as the most salient association. Therefore, when tested on subsequent days the mice retrieve the original fear memory and express fear at the cost of extinction because of the ambiguity of the immediate extinction contingency (fear memory most salient). This means that, despite subsequent extinction on test days, they continue to retrieve the fear state on future tests (resulting in spontaneous recovery) as the context and/or cues best predict the fear contingencies. This is akin to other theories that make explicit predictions about behavior when animals may not detect extinction contingencies (Capaldi 1966).

The ITC data support these theoretical mechanisms as their relative activation represented whether the context was a good, poor, or ambiguous predictor of shock. In the delayed groups, a test ambiguity model suggests that the long delay between acquisition or retrieval and extinction makes the extinction context no longer a good predictor of shock because there is strong temporal discrimination between the fearful state engaged by the acquisition/retrieval context and the nonreinforced extinction context. Indeed, the delayed group showed the strongest extinction and the most In:ImP activation. This suggests that when extinction contingencies are in effect, the In cluster (selectively associated with extinction contingencies) is strongly active relative to the more ambiguous ImP cluster. When the contingencies are ambiguous, such as in the immediate extinction groups, the fearful acquisition and retrieval states cannot be temporally discriminated from the extinction state (i.e., the proportion of In:ImP active neurons is 1:1), suggesting a more ambiguous activity state leading to default fear expression. However, when the context is a good predictor of shock (i.e., no extinction condition), a significantly lower In:ImP ratio is seen. Together, these data suggest that a high ratio of In:ImP neurons is indicative of contingencies associated with strong extinction retention, a neutral In:Imp ratio is indicative of ambiguous contingencies, and a high Imp:In ratio indicates that the context is a reliable predictor of shock. This fits well with our other amygdala data (CeL, CeM, BLA) and mPFC data (discussed above) as well as with recent physiological accounts suggesting that very specific neuronal groups are involved in predictive fear learning and expression (Palomares-Castillo et al. 2012; Pare and Duvarci 2012).
There is great promise for therapeutic strategies that place extinction-related therapy at the optimal temporal window following psychological trauma or trauma retrieval. However, the extension of our findings and findings like these to the clinic is premature. Although some studies in humans suggest that immediate behavioral intervention (e.g., extinction) may dampen fear and drug-seeking behavior (Schiller et al. 2008; Xue et al. 2012), there are human studies that indicate that this approach may have little effect or even enhance memory expression (Karpicke and Roediger 2008; Soeter and Kindt 2011; Wichert et al. 2011; Potts and Shanks 2012; Kindt and Soeter 2013). Particularly problematic for clinical applications are rodent and preclinical studies like ours suggesting that across a variety of conditions an immediate extinction experience may actually prevent the elimination or weakening of the fear response and promote its persistent expression (Morris et al. 2005; Chan et al. 2010; Kim et al. 2010). The idea that memories can be rewritten or erased by extinction is exciting from a theoretical and clinical standpoint, but the literature is mixed and suggests that the effects of extinction recency are better explained by associative and nonassociative learning accounts. Moreover, approaches that improve inhibitory learning may be beneficial to patients as they leave their memories and experiences intact while giving them the ability to learn from and cope with these powerful life events (Glannon 2006; Henry et al. 2007). Thus, advancing these basic findings into the clinic requires more work to determine the conditions and neural circuits that strengthen the learning that occurs during extinction without necessarily appealing to erasure mechanisms.

\section{Materials and Methods}

\section{Subjects}

A total of 282 male 8- to 12 -wk-old C57Bl/6J mice obtained from Jackson Laboratory or bred from mice originally obtained from Jackson were used in all experiments. Mice were maintained on a 12-h light/dark cycle with ad libitum access to food and water. Housing and experimental conditions were in accordance with OHSU IACUC and NIH "Principles of Laboratory Animal Care."

\section{Apparatus}

The context (Context A) consisted of a sound attenuated chamber illuminated with a house light. The chamber contained a circular Plexiglas arena placed on a grid floor through which a 2-s $0.35-\mathrm{mA}$ scrambled shock was delivered by a shock generator. Context A was cleaned with water prior to each session and had a fan running throughout the experiments to provide background noise. Context B (only used in Experiment 3) was an $18 \mathrm{~cm} \times 18 \mathrm{~cm}$ square Plexiglas chamber placed on a grid floor. Context B was kept dark and quiet (no fan), and was washed with $0.3 \%$ acetic acid. An infrared activity monitor fixed to the top of each chamber recorded freezing (Coulbourn Instruments). All chambers were housed in sound- and light-attenuating shells.

\section{General procedure}

Mice were handled for $30 \mathrm{sec}$ per day for $3 \mathrm{~d}$ prior to all experimental procedures. On acquisition day, mice were placed in the conditioning chamber and received four 2-sec $0.35-\mathrm{mA}$ shocks spaced at pseudo random 3-min intervals (except in Experiment 2). Animals were removed $30 \mathrm{sec}$ after the final shock for a total of $12 \mathrm{~min}$ of context exposure. Experimental timelines were staggered to ensure that, within each experiment, all groups were tested on the same days under common testing conditions. Each experiment was conducted in at least two replications of $n=4-$ 8 per group/replication. 


\section{Experiment 1. Effect of post-acquisition delay on extinction of fear expression and sensitivity to extinction}

\section{Effect of acquisition to extinction interval}

Mice received extinction (12-min context exposure) either immediately $(0 \mathrm{~h}, n=16), 1 \mathrm{~h}(n=8), 4 \mathrm{~h}(n=8)$, or $24 \mathrm{~h}$ following acquisition $(n=16)$. Previous research has found that pharmacological manipulations can affect memory consolidation at one or more of these intervals (Bourtchouladze et al. 1998; Monfils et al. 2009; Stafford and Lattal 2009). Each group was returned to their home cage and transported into a small procedure room between acquisition and extinction (mice in the 0-h group were in their home cages for $\sim 30-60 \mathrm{sec}$, enough time to clean the chambers and reset the computer program). The first test (12-min context exposure) was conducted immediately after extinction (ExtImm) and the next test was conducted $1 \mathrm{~d}$ later. Immediately following the 1-d test, mice were again tested for $12 \mathrm{~min}$ (1-d-Imm). To examine the effect of sensitivity of extinction strength, mice received either 3-min or 24-min extinction $0 \mathrm{~h}$ (immediate) or $24 \mathrm{~h}$ (delayed) following acquisition ( $n=8 /$ group). Testing occurred $1 \mathrm{~d}$ later.

\section{Experiment 2. Acquisition-extinction interval: Vulnerability to spontaneous recovery}

During acquisition, mice received a 3-min exposure to the context with a single 2 -sec $0.35-\mathrm{mA}$ shock at $2.5 \mathrm{~min}$. Subjects were removed and received 24-min extinction immediately (Imm) or $24 \mathrm{~h}(1 \mathrm{~d})$ following acquisition ( $n=16 /$ group). Mice were tested on four subsequent days following extinction (12-min context exposures). A retention test was conducted $14 \mathrm{~d}$ later.

\section{Experiment 3. Context processing in immediate extinction}

Following acquisition, mice received immediate extinction (24min context exposure) in Context A (Imm-A, $n=21$ ), a second group received immediate extinction (24-min context exposure) in Context B (Imm-B, $n=23)$, and a third group was handled while the others received extinction (No Ext, $n=16$ ). All groups were tested $1 \mathrm{~d}$ later in Context A and Context B with a 4-h interval separating these tests (counterbalanced exposure order).

\section{Experiment 4. Effect of post-retrieval delay on extinction of fear expression and sensitivity to extinction}

\section{Effect of retrieval-to-extinction interval}

All subjects received memory retrieval (3-min context exposure) $1 \mathrm{~d}$ following acquisition. Previous studies from our lab indicate that this retrieval duration followed by a protein synthesis inhibitor causes impairments in performance (Stafford and Lattal 2009). Following retrieval, mice were removed from the chambers, placed back into their home cage, and brought into an adjoining procedure room. They then received extinction (12-min context exposure) either immediately $(0 \mathrm{~h}), 1 \mathrm{~h}, 4 \mathrm{~h}$, or $24 \mathrm{~h}$ following retrieval ( $n=8$ /group). Testing (12-min context exposure) occurred $1 \mathrm{~d}$ and $14 \mathrm{~d}$ following extinction. To examine sensitivity to extinction session duration, mice received either 3-min or 24 -min extinction immediately $(0 \mathrm{~h})$ or $24 \mathrm{~h}$ following retrieval ( $n=8$ /group) and were tested at 1 and $14 \mathrm{~d}$ following extinction. To examine the role of retrieval, one group of mice received retrieval immediately followed by 24 -min extinction $(0 \mathrm{~h})$, a second group received 24-min extinction in the absence of retrieval ( 0 -h No Ret), a third group received retrieval followed $24 \mathrm{~h}$ later by 24-min extinction ( $24 \mathrm{~h}$ ), and a fourth group received 24-min extinction $24 \mathrm{~h}$ following the retrieval day in the absence of retrieval (0-h No Ret, all $n=7)$. Testing (12-min context exposure) occurred $1 \mathrm{~d}$ and $14 \mathrm{~d}$ following extinction.

\section{Experiment 5. Immunohistochemistry}

Behavioral procedures were similar to those of Experiments 2 and 4. Briefly, mice were separated into groups that received 24-min extinction either immediately or $24 \mathrm{~h}$ following fear acquisition or retrieval ( $n=8 /$ group). On the same day that these groups received extinction a separate group underwent acquisition with no extinction immediately $(n=8)$ to control for the presence of shock prior to sacrifice. Immunohistochemistry (IHC) for the immediate early gene c-Fos in select brain regions was examined in each group. Briefly, mice were sacrificed $30 \mathrm{~min}$ following extinction with brains subsequently fixed in formaldehyde and cryoprotected in sucrose. The No Ext group was sacrificed $1 \mathrm{~h}$ and $24 \mathrm{~min}$ following acquisition to equate the interval between behavior and sacrifice in the other groups. After sectioning 30- $\mu \mathrm{m}$ slices on a cryostat, IHC was performed on representative slices standardized to the same bregma level across brain regions (Bachtell et al. 1999; Stafford et al. 2012). Briefly, 0.3\% hydrogen peroxide was used to inhibit endogenous peroxide activity with blocking performed in 3\% goat serum. Slices were later incubated with antibody recognizing c-Fos (1:2000 dilution, Santa Cruz Biotechnology). The Vecstatin ABC kit (Vector Laboratory) and metal enhanced DAB kit (Pierce) was used for immunoreaction detection. Three slices per brain region were analyzed in all experiments with data (cell counts, see below) averaged per animal across slices.

\section{Distinction of ITC subpopulations}

We distinguished c-Fos ${ }^{+}$neurons in the main nucleus (In) and the medial paracapsular (ImP) using methods identical to those used by Busti et al. (2011) to evaluate Zif268 expression in these nuclei as well as in other studies of ITC populations (Hefner et al. 2008). Briefly, these nuclei were distinguished from other amygdalar nuclei as their activation patterns cluster together and the background staining produced by our IHC technique allows for distinction from other nuclei (Supplemental Fig. 3).

\section{In/ImP proportion analysis}

We calculated the proportion of c-Fos ${ }^{+}$In neurons to ImP neurons because: (1) Shock prior to c-Fos quantification led to significant activation of each of the individual ITC populations and obscured the main effect of extinction recency on these populations (to overcome the activation that masked these effects, we calculated a proportion score which normalized these substantially elevated activation patterns), and (2) reciprocal connections between the Imp and In clusters functionally inhibit one another. Indeed, it is the relative output of these nuclei that influences excitability within the CeL and CeM, ultimately impacting fear expression (i.e., ImP active during fear states and In active exclusively during low fear/extinction). Current theoretical accounts require that the relative effects of these distinct ITC masses are considered when evaluating their functional output. We therefore calculated the proportion of active In to ImP neurons to account for their combined role in mitigating the effect of extinction recency in a way consistent with current amygdala functional findings (Whittle et al. 2010; Busti et al. 2011; Manko et al. 2011; Palomares-Castillo et al. 2012; Pare and Duvarci 2012).

\section{Data analysis}

Fear was evaluated by measuring freezing behavior (absence of movement $\geq 3 \mathrm{sec}$ ) using the infrared activity monitors. Freezing was analyzed in 3-min blocks in all sessions. In all experiments there was no difference between groups during acquisition (all $P>0.1$ ). Due to rapid within-session extinction during the test sessions, data during the first 3-min were analyzed for differences between groups (Lattal and Abel 2004; Stafford et al. 2012). Quantification of c-Fos was performed by counting c-Fos positive nuclei in each brain region by an experimenter blinded to experimental conditions. Group differences were analyzed with analysis of variance (ANOVA). Simple planned post-hoc comparisons were tested using a Fisher's LSD. For all statistical tests the $\alpha$ was set $\leq 0.05$. 


\section{Acknowledgments}

We thank William Giardino for helpful comments on the manuscript. This work was supported by National Institutes of Health grant R01MH077111 to K.M.L. as well as Vertex Pharmaceutical Scholarship and F31MH087031 to J.M.S.

\section{References}

Bachtell RK, Wang YM, Freeman P, Risinger FO, Ryabinin AE. 1999. Alcohol drinking produces brain region-selective changes in expression of inducible transcription factors. Brain Res 847: 157-165.

Bourtchouladze R, Abel T, Berman N, Gordon R, Lapidus K, Kandel ER. 1998. Different training procedures recruit either one or two critical periods for contextual memory consolidation, each of which requires protein synthesis and PKA. Learn Mem 5: 365-374.

Bouton ME. 2004. Context and behavioral processes in extinction. Learn Mem 11: $485-494$.

Brandon SE, Vogel EH, Wagner AR. 2003. Stimulus representation in SOP: I. Theoretical rationalization and some implications. Behav Process 62: $5-25$.

Busti D, Geracitano R, Whittle N, Dalezios Y, Manko M, Kaufmann W, Sätzler K, Singewald N, Capogna M, Ferraguti F. 2011. Different fear states engage distinct networks within the intercalated cell clusters of the amygdala. J Neurosci 31: 5131-5144.

Capaldi EJ. 1966. Partial reinforcement: A hypothesis of sequential effects. Psychol Rev 73: 459-477.

Chan WY, Leung HT, Westbrook RF, McNally GP. 2010. Effects of recent exposure to a conditioned stimulus on extinction of Pavlovian fear conditioning. Learn Mem 17: 512-521.

Ciocchi S, Herry C, Grenier F, Wolff SB, Letzkus JJ, Vlachos I, Ehrlich I, Sprengel R, Deisseroth K, Stadler MB, et al. 2010. Encoding of conditioned fear in central amygdala inhibitory circuits. Nature 468: 277-282.

Clem RL, Huganir RL. 2010. Calcium-permeable AMPA receptor dynamics mediate fear memory erasure. Science 330: 1108-1112.

Costanzi M, Cannas S, Saraulli D, Rossi-Arnaud C, Cestari V. 2011. Extinction after retrieval: Effects on the associative and nonassociative components of remote contextual fear memory. Learn Mem 18: 508-518.

Delamater AR. 2004. Experimental extinction in Pavlovian conditioning: Behavioural and neuroscience perspectives. QJ Exp Psychol B 57: 97-132.

Dobi A, Sartori SB, Busti D, Van der Putten H, Singewald N, Shigemoto R, Ferraguti F. 2013. Neural substrates for the distinct effects of presynaptic group III metabotropic glutamate receptors on extinction of contextual fear conditioning in mice. Neuropharmacology 66: 274-289.

Flavell CR, Barber DJ, Lee JL. 2011. Behavioural memory reconsolidation of food and fear memories. Nat Commun 2: 504.

Glannon W. 2006. Psychopharmacology and memory. J Med Ethics 32: $74-78$.

Gleitman H, Jung L. 1963. Retention in rats: The effect of proactive interference. Science 142: 1683-1684.

Haubensak W, Kunwar PS, Cai H, Ciocchi S, Wall NR, Ponnusamy R, Biag J, Dong HW, Deisseroth K, Callaway EM, et al. 2010. Genetic dissection of an amygdala microcircuit that gates conditioned fear. Nature 468 : $270-276$.

Hefner K, Whittle N, Juhasz J, Norcross M, Karlsson RM, Saksida LM, Bussey TJ, Singewald N, Holmes A. 2008. Impaired fear extinction learning and cortico-amygdala circuit abnormalities in a common genetic mouse strain. J Neurosci 28: 8074-8085.

Henry M, Fishman JR, Youngner SJ. 2007. Propranolol and the prevention of post-traumatic stress disorder: Is it wrong to erase the "sting" of bad memories? Am J Bioeth 7: 12-20.

Hui IR, Hui GK, Roozendaal B, McGaugh JL, Weinberger NM. 2006 Posttraining handling facilitates memory for auditory-cue fear conditioning in rats. Neurobiol Learn Mem 86: 160-163.

Ishii D, Matsuzawa D, Matsuda S, Tomizawa H, Sutoh C, Shimizu E. 2012 No erasure effect of retrieval-extinction trial on fear memory in the hippocampus-independent and dependent paradigms. Neurosci Lett 523: $76-81$.

Johnson JS, Escobar M, Kimble WL. 2010. Long-term maintenance of immediate or delayed extinction is determined by the extinction-test interval. Learn Mem 17: 639-644.

Karpicke JD, Roediger HL III. 2008. The critical importance of retrieval for learning. Science 319: 966-968.

Kim SC, Jo YS, Kim IH, Kim H, Choi JS. 2010. Lack of medial prefrontal cortex activation underlies the immediate extinction deficit. J Neurosci 30: $832-837$

Kindt M, Soeter M. 2013. Reconsolidation in a human fear conditioning study: A test of extinction as updating mechanism. Biol Psychol 92: 43-50.
Lattal KM, Abel T. 2004. Behavioral impairments caused by injections of the protein synthesis inhibitor anisomycin after contextual retrieval reverse with time. Proc Natl Acad Sci 101: 4667-4672.

Li G, Amano T, Pare D, Nair SS. 2011. Impact of infralimbic inputs on intercalated amygdala neurons: A biophysical modeling study. Learn Mem 18: 226-240.

Likhtik E, Popa D, Apergis-Schoute J, Fidacaro GA, Pare D. 2008. Amygdala intercalated neurons are required for expression of fear extinction. Nature 454: 642-645.

Manko M, Geracitano R, Capogna M. 2011. Functional connectivity of the main intercalated nucleus of the mouse amygdala. J Physiol 589: 1911-1925.

Mao SC, Hsiao YH, Gean PW. 2006. Extinction training in conjunction with a partial agonist of the glycine site on the NMDA receptor erases memory trace. J Neurosci 26: 8892-8899.

Maren S, Chang CH. 2006. Recent fear is resistant to extinction. Proc Natl Acad Sci 103: 18020-18025.

McGaugh JL. 2006. Make mild moments memorable: Add a little arousal. Trends Cogn Sci 10: 345-347.

McGaugh JL, Roozendaal B. 2009. Drug enhancement of memory consolidation: Historical perspective and neurobiological implications. Psychopharmacology (Berl) 202: 3-14.

McIntyre CK, McGaugh JL, Williams CL. 2012. Interacting brain systems modulate memory consolidation. Neurosci Biobehav Rev 36: $1750-1762$.

Monfils MH, Cowansage KK, Klann E, Ledoux JE. 2009. Extinction-reconsolidation boundaries: Key to persistent attenuation of fear memories. Science 324: 951-955.

Morris RW, Furlong TM, Westbrook RF. 2005. Recent exposure to a dangerous context impairs extinction and reinstates lost fear reactions. J Exp Psychol Anim Behav Process 31: 40-55.

Myers KM, Ressler KJ, Davis M. 2006. Different mechanisms of fear extinction dependent on length of time since fear acquisition. Learn Mem 13: $216-223$

Norrholm SD, Vervliet B, Jovanovic T, Boshoven W, Myers KM, Davis M, Rothbaum B, Duncan EJ. 2008. Timing of extinction relative to acquisition: A parametric analysis of fear extinction in humans. Behav Neurosci 122: 1016-1030.

Palomares-Castillo E, Hernandez-Perez OR, Perez-Carrera D, Crespo-Ramirez M, Fuxe K, Perez de la Mora M. 2012. The intercalated paracapsular islands as a module for integration of signals regulating anxiety in the amygdala. Brain Res 1476: $211-234$.

Pare D, Duvarci S. 2012. Amygdala microcircuits mediating fear expression and extinction. Curr Opin Neurobiol 22: 717-723.

Pavlov IP. 1927. Conditioned reflexes, an investigation of the physiological activity of the cerebral cortex, Oxford University Press, London.

Potts R, Shanks DR. 2012. Can testing immunize memories against interference? J Exp Psychol Learn Mem Cogn 38: 1780-1785.

Quirk GJ, Mueller D. 2008. Neural mechanisms of extinction learning and retrieval. Neuropsychopharmacology 33: 56-72.

Rao-Ruiz P, Rotaru DC, van der Loo RJ, Mansvelder HD, Stiedl O, Smit AB, Spijker S. 2011. Retrieval-specific endocytosis of GluA2-AMPARs underlies adaptive reconsolidation of contextual fear. Nat Neurosci 14: $1302-1308$.

Redish AD, Jensen S, Johnson A, Kurth-Nelson Z. 2007. Reconciling reinforcement learning models with behavioral extinction and renewal: Implications for addiction, relapse, and problem gambling. Psychol Rev 114: 784-805.

Schiller D, Levy I, Niv Y, LeDoux JE, Phelps EA. 2008. From fear to safety and back: Reversal of fear in the human brain. J Neurosci $\mathbf{2 8}$ : 11517-11525.

Soeter M, Kindt M. 2011. Disrupting reconsolidation: Pharmacological and behavioral manipulations. Learn Mem 18: 357-366.

Stafford JM, Lattal KM. 2009. Direct comparisons of the size and persistence of anisomycin-induced consolidation and reconsolidation deficits. Learn Mem 16: 494-503.

Stafford JM, Raybuck JD, Ryabinin AE, Lattal KM. 2012. Increasing histone acetylation in the hippocampus-infralimbic network enhances fear extinction. Biol Psychiatry 72: 25-33.

Whittle N, Hauschild M, Lubec G, Holmes A, Singewald N. 2010. Rescue of impaired fear extinction and normalization of cortico-amygdala circuit dysfunction in a genetic mouse model by dietary zinc restriction. $J$ Neurosci 30: 13586-13596.

Wichert S, Wolf OT, Schwabe L. 2011. Reactivation, interference, and reconsolidation: Are recent and remote memories likewise susceptible? Behav Neurosci 125: 699-704.

Xue YX, Luo YX, Wu P, Shi HS, Xue LF, Chen C, Zhu WL, Ding ZB, Bao YP, Shi J, et al. 2012. A memory retrieval-extinction procedure to prevent drug craving and relapse. Science 336: 241-245.

Received November 30, 2012; accepted in revised form December 20, 2012. 


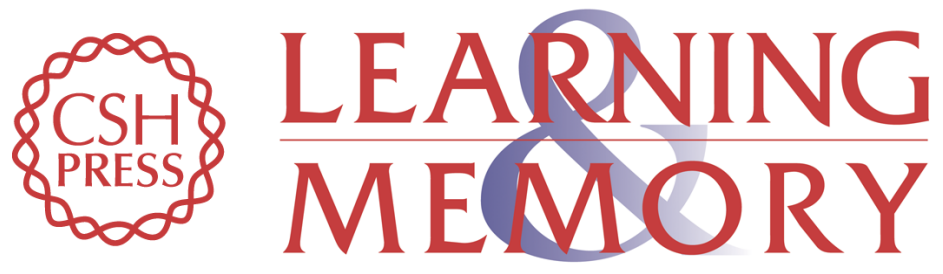

\section{Exposure to a fearful context during periods of memory plasticity impairs extinction via hyperactivation of frontal-amygdalar circuits}

James M. Stafford, DeeAnna K. Maughan, Elena C. Ilioi, et al.

Learn. Mem. 2013, 20:

Access the most recent version at doi:10.1101/lm.029801.112

Supplemental http://learnmem.cshlp.org/content/suppl/2013/02/19/20.3.156.DC1
Material

References This article cites 50 articles, 23 of which can be accessed free at:

http://learnmem.cshlp.org/content/20/3/156.full.html\#ref-list-1

License

Email Alerting Receive free email alerts when new articles cite this article - sign up in the box at the Service top right corner of the article or click here. 